Proc. XII Int. School on Theoretical Physics - Symmetry and Structural Properties of Condensed Matter

\title{
Comparison of Methods for Determining the Contrast Distribution in Interference Images with Speckle Noise
}

\author{
L. PYZIAK ${ }^{a, *}$, M.J. MATCZAK ${ }^{b}$ AND P. ZIEBA ${ }^{c}$ \\ ${ }^{a}$ Faculty of Mathematics and Applied Physics, Rzeszów University of Technology, \\ al. Powstańców Warszawy 12, 35-959 Rzeszów, Poland \\ ${ }^{b}$ Interfaculty Division of Mathematics and Natural Sciences, The State Higher School of Technology and Economics, \\ S. Czarnieckiego 16, 37-500 Jarosław, Poland \\ ${ }^{c}$ Faculty of Mathematics and Natural Sciences, University of Rzeszów, S. Pigonia 1, 35-310 Rzeszów, Poland

\begin{abstract}
Information about certain physical states of an examined object is encoded in the distribution of the fringe
\end{abstract} \\ contrast in the metrological interference images of the object. Determining the contrast is one of the steps of \\ converting the image into the distribution of the measured quantity in holographic fringe-contrast interferometry. \\ In this paper, three methods of determining the fringe contrast in interference images containing speckle noise are \\ compared. As the criterion for comparing the methods, the mean absolute error has been used. It turns out, in \\ accordance with this criterium, the most effective method is the phase-shifting one.
}

DOI: 10.12693/APhysPolA.132.173

PACS/topics: $42.25 . \mathrm{Hz}, 42.30 .-\mathrm{d}, 42.30 . \mathrm{Kq}, 42.30 . \mathrm{Ms}$

\section{Introduction}

The distribution of the fringe contrast $V(x, y)$ is the carrier and the source of information used in the fringecontrast interferometry $[1,2]$, whose recorded images are characterized by the following distribution of the light intensity [2]:

$$
\begin{aligned}
& I(x, y)=I_{0}(x, y)[1+V(x, y) \cos \varphi(x, y)] \\
& \quad+N_{I}(x, y),
\end{aligned}
$$

where $I_{0}(x, y)$ is the light intensity in a non-interferential image of an object, $\varphi(x, y)$ is the phase difference distribution of interfering beams, and $N_{I}(x, y)$ is the component determined by stochastic speckle noise $[3,4]$. The speckle noise can be diminished by means of optical methods $[4,5]$ as well as numerical ones $[6-8]$, but it cannot be totally eliminated. Determining the contrast distribution is one of the main stages of transforming the images obtained by the optical methods into the distribution of a given measured quantity, described by a scalar, vector, or tensor field. The purpose of the paper is to compare three methods for determining the contrast distribution. These methods have their origin in three different processes based on:

- classical definition of fringe contrast - definitional method (DM),

- filtration in the spatial frequency domain - the Fourier method (FM),

- shifting the phase difference between interfering beams - phase-shifting method (PSM).

\footnotetext{
*corresponding author; e-mail: 1.pyziak@prz.edu.pl
}

Generation of interference images - which are the main subject of this study - as well as determining the contrast distribution have been carried out in Mathematica environment.

\section{Methods of determining the fringe-contrast distribution \\ 2.1. Definitional method}

On the basis of methods related to determining the interference-phase distribution $[9,10]$, the method of determining the contrast was proposed [11]. It is based on the contrast definition:

$$
V=\frac{I_{\max }-I_{\min }}{I_{\max }+I_{\min }}
$$

where $I_{\max }$ and $I_{\min }$ are the maximal and minimal values of the light intensity in the vicinity of a considered point, respectively. The procedure of determining the contrast requires several steps.

Due to large contrast and dynamics of the speckle noise, first step of the procedure is its reduction. In this paper, the averaging of the interference images of the same distribution of fringes and their contrast, but different speckle-noise distribution, has been used. Figure 1a shows an exemplary distribution of the interference fringes after this step, while single interference image with speckle noise has the form shown in Fig. 2a. Skeletonization of fringes that determines the local maxima and minima of the interference-fringes intensity distribution [11] is the second step of the procedure. The next step is the calculation of contrast values at the lines lying halfway between the extreme lines. The contrast value is determined according to the formula (2). In the last step, as a result of interpolation process, the contrast distribution in the entire interference image is obtained (Fig. 1b). 

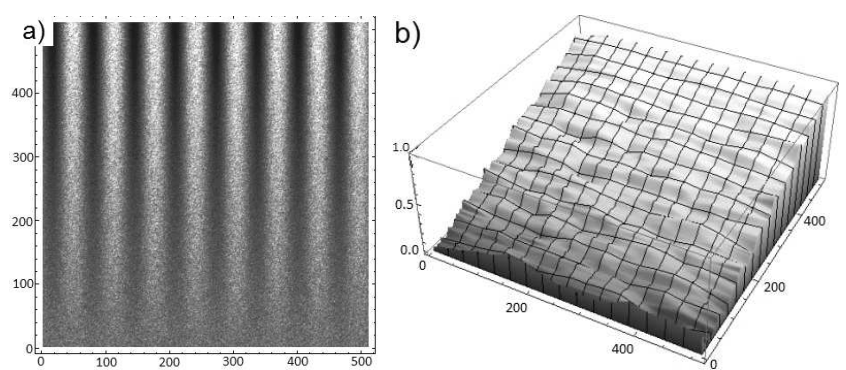

Fig. 1. (a) An exemplary interference image after speckle-noise reduction, (b) contrast distribution in the interference image.

\subsection{Fourier method}

The method based on the filtration of the Fourier spectrum was proposed in [2]. This approach assumes separation of the spectral ranges of the individual components of (1), which is not exactly fulfilled in real conditions. In the first step of the procedure, it is necessary to remove the spectrum corresponding to the last term of the expression (1), namely the high-frequency component $N_{I}(x, y)$. After carrying out such a filtration, and then the inversion of the Fourier transformation, the obtained intensity distribution becomes

$$
I_{1}(x, y)=I_{0}(x, y)[1+V(x, y) \cos (\varphi(x, y))] .
$$

Next, in order to calculate the light intensity distribution in a non-interferential image of an object, a low pass filter is applied. Then

$$
I_{2}(x, y)=I_{0}(x, y) \text {. }
$$

Dividing the obtained distributions one by the other we get the distribution described by the formula

$$
I_{3}(x, y)=1+V(x, y) \cos (\varphi(x, y)) .
$$

Then, taking into account the fact that the spectrum of such distributions is Hermitian [14], by cutting off halfplane of spatial frequencies and the zero frequency, we obtain the complex distribution described as

$$
I_{4}(x, y)=V(x, y) \mathrm{e}^{\mathrm{i} \varphi(x, y)} .
$$

The calculation of the absolute value of this distribution will determine the distribution of the fringe contrast.

\subsection{Phase-shifting method}

Many algorithms for determining the phase distribution based on the phase-shifting method can be found in scientific literature. Some of them have been adapted to determine the contrast distribution [10]. One of the classic algorithms is the four-image algorithm, for which the phase step increment is equal to $\Delta \alpha=\frac{\pi}{4}$. In this algorithm, the distribution intensity of each of the four interference images $I_{i}(x, y)$ with the phase shift, $(i-1) \Delta \alpha$, $i \in\{1,2,3,4\}$ can be described according to the formula [10]:

$$
\begin{gathered}
I_{i}(x, y)=I_{0}(x, y)[1+V(x, y) \times \\
\quad \times \cos (\varphi(x, y)+(i-1) \Delta \alpha)] .
\end{gathered}
$$

The formula that enables us to calculate the contrast distribution based on the four interference images, which are described by the Eq. (7), takes the form [10]:

$$
\begin{aligned}
& V(x, y)=\left\{\left[I_{4}(x, y)-I_{2}(x, y)\right]^{2}\right. \\
& \left.\quad+\left[I_{1}(x, y)-I_{3}(x, y)\right]^{2}\right\}^{1 / 2} /\left(2 I_{0}\right) .
\end{aligned}
$$

\section{Comparison methodology}

Interference images, on which the comparative study of the selected contrast determination methods has been carried out, was numerically generated. It was assumed the linear change of the contrast, where the direction of the change in one test image is parallel to interference fringes (Fig. 2a) while in the second one it is normal to them (Fig. 2b). Speckle noise was introduced into these test images with the use of the algorithm, which is described in [4].
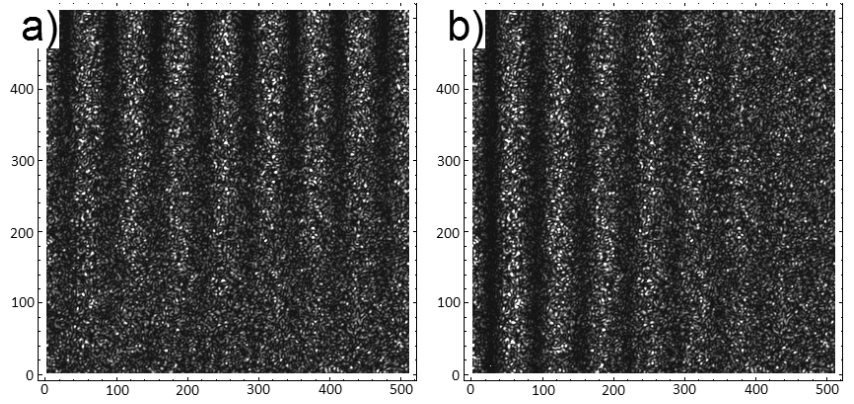

Fig. 2. Interference images with speckle noise and with vertical (a) and horizontal (b) change of the contrast.

The mean speckle size in all images is equal to 6 pixels, which gives about 10.7 speckles per the width of one interference fringe. The images have been subject to 8-bit quantization. As the method for reduction of the speckle noise, the averaging of the speckle distribution over its $N$ realizations was assumed according to the formula

$$
\bar{I}(i, j)=\frac{1}{N} \sum_{n=1}^{N} I_{n}(i, j)
$$

where $n$ denotes the index of the particular realization. The particular images differ amongst themselves only in the structure of the speckle distribution.

In this work, we assumed the average absolute error as criteria of comparing the methods of determining the fringe-contrast distribution in interference images. The average absolute error is described by

$$
\delta V=\frac{1}{N_{x} N_{y}} \sum_{i=1}^{N_{x}} \sum_{j=1}^{N_{y}}\left|V_{w}(i, j)-V(i, j)\right|,
$$

where $N_{x}, N_{y}$ - the size of the image in pixels, $i, j-$ coordinates of pixels, $V_{w}, V$ the contrast values determined by a particular method and generated in source images, respectively. The mean absolute error $\delta V$ indicates the average deviation of the values $V_{w}, V$. 


\section{Results}

The plots in Fig. 3 show the dependences of the assumed criteria for the accuracy of determining contrast distribution on the number of images taken into account in averaging, for two test images shown in Fig. 2. In both plots, the value $\delta V$ is decreasing very quickly with increase of the number of images taken in averaging for all examined methods, but the fastest fall is in the case of the phase-shifting method. In this case, for $N=20$ the error value reaches the level that does not significantly decrease when the number $N$ increases further. For other methods, it is necessary to average more interference images. However, ultimately, the outcomes of all methods give very similar results.

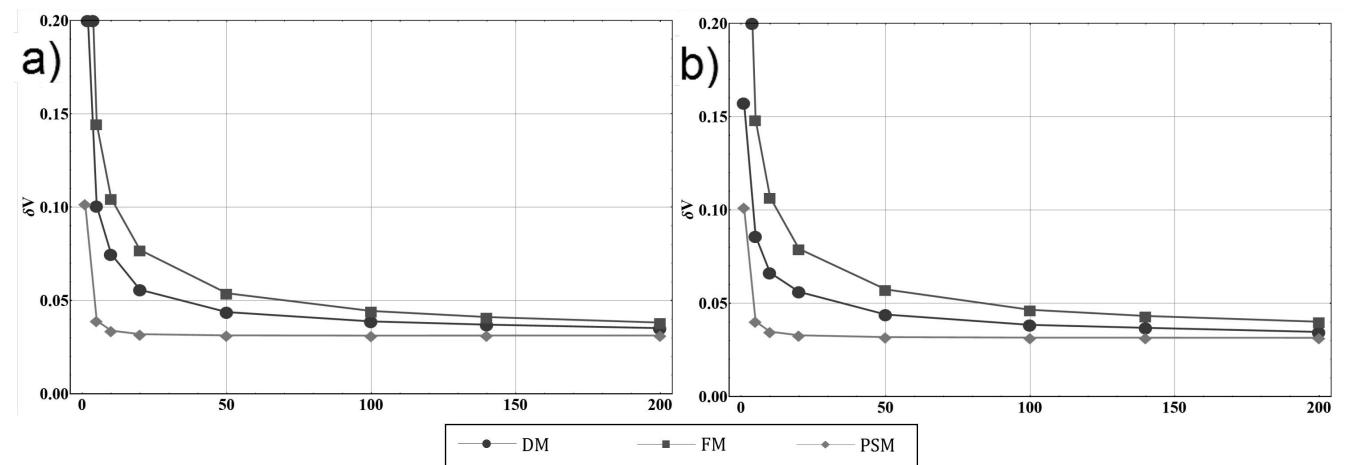

Fig. 3. The dependence of the absolute error on $N$, where the curves have been obtained for different methods (DM, FM, and PSM). The results have been obtained for two test interference images: (a) Fig. 2a and (b) Fig. 2b.

The results of our work show that whatever method is used for determining the contrast distribution, the errors are almost the same and do not depend on the direction of contrast changes. It is also worth noting that despite the increase in the number of images taken for averaging, even for the best method, the errors do not reach zero value, but tend to the asymptote, which in the case of the absolute error is approximately equal to 0.03. According to the authors, this asymptotic error originates mainly from the averaging process of the speckle distributions. The process of averaging leads to the modifications of extreme values of contrast in the image with respect to the original contrast distribution.

\section{Conclusions}

In this paper, we compared three methods for determining the contrast distribution of interference fringes using the images with the speckle noise. From the point of view of the adopted criteria, the most effective method has been proved to be the method based on the phase shift introduced into the reference beam. This method, in comparison to the others, is resistant to the noise.

\section{References}

[1] M.J. Matczak, Proc. SPIE 370, 163 (1982).

[2] M.J. Matczak, Opt. Lasers Eng. 9, 121 (1988).
[3] Laser Speckle and Related Phenomena, Topics in Applied Optics, Ed. C.J. Dainty, Springer, Berlin 1975.

[4] J.W. Goodman, Speckle Phenomena in Optics: Theory and Applications, Roberts and Co., Englewood 2007.

[5] M. Matsumura, Appl. Opt. 14, 660 (1975).

[6] E. Bieber, W. Osten, Proc. SPIE 1121, 393 (1989).

[7] A. Ochoa, A.A. Silva-Moreno, Opt. Commun. 270, 161 (2007).

[8] B. Zielinski, K. Patorski, Opto-Electron. Rev. 18, 155 (2010).

[9] T. Kreis, Handbook of Holographic Interferometry. Optical and Digital Methods, Wiley, Weinheim 2005.

[10] D.Y. Robinson, G.S. Reid, Interferogram Analysis: Digital Fringe Pattern Measurement Technique, Institute of Physics Publ., Bristol 1993.

[11] M.J. Matczak, Proc. SPIE 661, 280 (1986).

[12] J.W. Goodman, Statistical Optics, Wiley, New York 2000.

[13] M.J. Matczak, J. Budziński, Proc. SPIE 1121, 136 (1989).

[14] K. Gniadek, Optical Information Processing, PWN, Warsaw 1992

[15] K. Patorski, M. Kujawińska, L. Sałbut, Laser Interferometry with Automatic Image Analysis, Warsaw University of Technology, Warsaw 2005. 